\title{
Decreased susceptibility of Neisseria gonorrhoeae isolates from Switzerland to Cefixime and Ceftriaxone: antimicrobial susceptibility data from 1990 and 2000 to 2012
}

\author{
Helen Kovari ${ }^{*}$, Maria DG de Melo Oliveira ${ }^{2}$, Paula Hauser ${ }^{1}$, Severin Läuchli ${ }^{3}$, Jürg Meyer ${ }^{3}$, Rainer Weber ${ }^{1}$
} and Reinhard Zbinden²

\begin{abstract}
Background: Neisseria gonorrhoeae can rapidly develop resistance to antimicrobial agents. Over the last years, decreased gonococcal susceptibility to third-generation cephalosporins, especially cefixime, emerged worldwide. Therefore, current international guidelines recommend dual therapy for gonorrhoea with ceftriaxone plus either azithromycin or doxycycline. Gonococcal susceptibility data in Switzerland are sparse.

Methods: We investigated the prevalence of antibiotic susceptibility of $N$. gonorrhoeae in specimens collected between 1990 and 2012 at the University of Zurich, Switzerland. Minimum inhibitory concentrations (MICs) for cefixime, ceftriaxone, ciprofloxacin, and penicillin were determined by Etests. The European Committee on Antimicrobial Susceptibility Testing (EUCAST) breakpoints were used to define reduced susceptibility.

Results: A total of 320 isolates were tested. Between 1990 and 2006 all tested samples were susceptible to both cephalosporins. Subsequently, the prevalence of elevated MICs for cefixime increased to 10.4\% (2007/2008), 11.5\% (2009/2010), and 11.4\% (2011/2012); and for ceftriaxone to 2.4\% (2007/2008), 4.7\% (2009/2010), and 0\% (2011/2012), respectively. The prevalence of resistance to ciprofloxacin (72.7\%) and penicillin (22.7\%) was high in 2011/2012.

Conclusions: Decreasing susceptibility of N. gonorrhoeae to third-generation cephalosporins in Switzerland supports treatment recommendations with ceftriaxone plus azithromycin or doxycycline. Health-care providers need to be aware of possible treatment failures with cephalosporins. Continued surveillance of gonococcal antimicrobial resistance is essential.
\end{abstract}

Keywords: Neisseria gonorrhoeae, Gonorrhoea, Antimicrobial resistance, Cephalosporins

\section{Background}

Infections with Neisseria gonorrhoeae may cause serious complications, including pelvic inflammatory disease, infertility, peritonitis, and chronic pain, and it enhances HIV transmission [1,2]. Effective treatment of gonorrhoea is mandatory to control disease transmission and prevent sequelae. However, N. gonorrhoeae rapidly developed antimicrobial resistance to all previously recommended first-line drugs, including penicillins, tetracyclines, and

\footnotetext{
*Correspondence: helen.kovari@usz.ch

1 Division of Infectious Diseases and Hospital Epidemiology, University Hospital Zurich, University of Zurich, Zurich, Switzerland

Full list of author information is available at the end of the article
}

fluoroquinolones. Third-generation cephalosporins, such as cefixime and ceftriaxone, remain the only readily available active antimicrobial class. In recent years, however, gonococcal strains with reduced susceptibility to cefixime emerged and spread worldwide [3]. Clinical treatment failures with cefixime have been reported in Japan, several European countries, Canada, and South Africa [4-10]. In 2009, the first treatment failures to ceftriaxone were observed in Australia [11] and 2010 in Sweden [12]. Currently, the identification of extensively drug-resistant (XDR) N. gonorrhoeae strains with high-level ceftriaxone resistance in Japan [13], France [14], and Spain [15], are of major concern. In addition to high ceftriaxone minimum 
inhibitory concentrations (MICs), these XDR strains are typically multidrug-resistant, exhibiting additional resistance to fluoroquinolones as well as to older drugs. Consequently, most treatment guidelines now recommend combination therapy with the injectable agent ceftriaxone plus either azithromycin or doxycycline instead of oral monotherapy with cefixime $[16,17]$.

In Switzerland, a strong rise in N. gonorrhoeae infections was observed during the past decade. Notifications have increased nearly threefold, with 521 reported cases in 2003, and 1569 cases in 2012, of which 77\% occurred in men [18]. Reporting gonorrhoea to public health authorities has been mandatory in Switzerland since 1988. However, gonococcal susceptibility data in Switzerland are sparse [19]. Due to the current $N$. gonorrhoeae resistance problem, improving gonococcal antimicrobial resistance surveillance is of utmost importance and high on the agenda of the WHO's "Global action plan to control the spread and impact of antimicrobial resistance in Neisseria gonorrhoeae, 2012" [20]. Local antimicrobial surveillance is essential for evidence-based treatment recommendations. Given the lack of data, we investigated the distribution of antibiotic susceptibility of $N$. gonorrhoeae in specimens collected between 1990 and 2012.

\section{Methods}

All isolates were obtained in the northeastern part of Switzerland and the region of Zurich between January 1990 and December 2012. Specimens were cultured from symptomatic gonorrhoea patients with only one isolate taken per patient in case they were infected at multiple sites. Species confirmation and susceptibility testing was performed at the Institute of Medical Microbiology, University of Zurich. Specimens were cultured on selective media, i.e. until 2009 on Thayer Martin agar (Difco; Becton, Dickinson and Company, Basel, Switzerland), supplemented with IsoVitalex, and after 2009 on VCA3 agar (BioMérieux, Marcy-l'Etoile, France). The commercial biochemical gallery Api NH (BioMérieux) was used for the identification.

Minimum inhibitory concentrations (MICs) for cefixime, ceftriaxone, ciprofloxacin and penicillin were determined by the Etest method (BioMérieux, Marcy l'Etoile, France) on Difco GC agar until 2004 (Becton Dickinson, Cockeyswille, MD, USA) and after 2005 on chocolate agar with PolyViteX (BioMérieux) or on MH-horse blood agar (BioMérieux). In order to demonstrate the comparability of the different methods, we tested 9 isolates (1990-2004) and the reference ATCC strain 49226 with all three media for all four antibiotics (Additional file 1: Table S1). Beta-lactamase test was performed with nitrocefin (Becton Dickinson). The European Committee on Antimicrobial Susceptibility Testing (EUCAST) breakpoints were used [21]: Breakpoints for the definition of decreased cefixime and ceftriaxone susceptibility were MICs $>0.125 \mathrm{mg} / \mathrm{L}$, and ciprofloxacin resistance was defined by MICs $>0.064 \mathrm{mg} / \mathrm{L}$. Penicillin resistance was defined by either MICs $>1.0 \mathrm{mg} / \mathrm{L}$ or the presence of penicillinase-producing strains.

The following data was available for all isolates collected between 2005 and 2012: specimen source, date specimen obtained, sex, age, and specimen site. For all isolates collected before 2005 only date of specimen collection was available. The study was approved by the ethics committee of Zurich (ethics approval number: KEK-ZH-Nr. 2012-0510).

\section{Results}

A total of 318 isolates from the region of Zurich and the northeastern part of Switzerland were analysed, including 42 samples from 1990, 66 from 2000 to 2004, and 210 samples from 2005 to 2012. The level of coverage (number of isolates tested compared to the number of reported cases in the region of Zurich) was 5\%. Of the samples collected between 2005 and 2012, 168 (79.3\%) were collected from men, 35 (16.5\%) from women, and in $9(4.2 \%)$ samples data on sex and age was missing. Median age for males was 35 years (range 17 to 86 years) and for females 31 years (range 16 to 66 years). The isolates were obtained from the urethra (60\%), cervix (13\%), other locations (4\%), including anorectum, pharynx, joint and eye, or from unknown sites (24\%).

The results of antimicrobial susceptibilities are summarized in Table 1. No decreased susceptibility to cefixime was detected until 2006 in the tested strains, whereas 2007/2008 10.4\%, 2009/2010 11.5\%, and 2011/2012 11.4\% of isolates displayed in vitro resistance to cefixime. In Figure 1 cefixime MIC distribution between 2005 and 2012 is illustrated. From 2005 onwards, isolates demonstrate a clear shift towards higher MIC categories.

Ceftriaxone susceptibility was $100 \%$ until 2007 . Between 2008 and 2010 four isolates exhibited decreased susceptibility to ceftriaxone, with one isolate from 2008 showing a ceftriaxone MIC of $0.25 \mathrm{mg} / \mathrm{L}$. In three of these patients the site of infection was urethral and in one patient anorectal. Most strains showed low ceftriaxone MIC's of $\leq 0.016 \mathrm{mg} / \mathrm{L}$ between 2005 and 2012. However, the proportion of isolates in higher MIC categories has clearly increased in recent years (Figure 2).

Fluoroquinolone-resistant $N$. gonorrhoeae emerged in Switzerland during the 1990s, with a ciprofloxacin resistance rate of $2.4 \%$ in 1990 . In the early 2000 s, a rapid increase of ciprofloxacin resistance from $30.0 \%$ in 2001/ 2002 to $72.7 \%$ in 2011/2012 was observed (Table 1). Penicillin resistance rate was $22.7 \%$ in $2011 / 2012$. However, only $18.2 \%$ of the strains were fully penicillin susceptible according to EUCAST breakpoints $(\leq 0.06 \mathrm{mg} / \mathrm{L})$. 
Table $1 \mathrm{~N}$. gonorrhoeae with decreased susceptibility* to cefixime, ceftriaxone, and resistance to ciprofloxacin and penicillin in Switzerland, 1990-2012

\begin{tabular}{|c|c|c|c|c|c|c|c|c|c|c|c|c|}
\hline \multirow[b]{2}{*}{ Year } & \multicolumn{3}{|c|}{ Cefixime } & \multicolumn{3}{|c|}{ Ceftriaxone } & \multicolumn{3}{|c|}{ Ciprofloxacin } & \multicolumn{3}{|c|}{ Penicillin } \\
\hline & Tested, $\mathbf{n}^{\#}$ & DS, n (\%) & $(95 \% \mathrm{Cl})$ & Tested, $\mathbf{n}^{\#}$ & DS, n (\%) & $(95 \% \mathrm{Cl})$ & Tested, $\mathbf{n}^{\#}$ & $\mathrm{R}, \mathrm{n}(\%)$ & $(95 \% \mathrm{Cl})$ & Tested, $\mathbf{n}^{\#}$ & $\mathrm{R}, \mathrm{n}(\%)$ & $(95 \% \mathrm{Cl})$ \\
\hline 1990 & 16 & $0(0)$ & $(0-21)$ & 16 & $0(0)$ & $(0-21)$ & 42 & $1(2.4)$ & $(0-12)$ & 42 & $9(21.4)$ & $(10-37)$ \\
\hline 2000-2002 & 7 & $0(0)$ & $(0-40)$ & 7 & $0(0)$ & $(0-40)$ & 33 & $10(30.3)$ & (15-48) & 33 & $4(12.1)$ & $(3-28)$ \\
\hline $2003 / 2004$ & 10 & $0(0)$ & $(0-30)$ & 10 & $0(0)$ & $(0-30)$ & 33 & $16(48.5)$ & $(31-66)$ & 33 & $13(39.4)$ & $(23-58)$ \\
\hline $2005 / 2006$ & 17 & $0(0)$ & $(0-19)$ & 32 & $0(0)$ & $(0-11)$ & 36 & $21(58.3)$ & $(41-74)$ & 31 & $7(22.6)$ & $(10-41)$ \\
\hline $2007 / 2008$ & 48 & $5(10.4)$ & $(3.4-22)$ & 85 & $2(2.4)$ & $(0.3-8)$ & 85 & $62(72.9)$ & $(62-82)$ & 84 & $25(29.8)$ & $(20-41)$ \\
\hline $2009 / 2010$ & 26 & $3(11.5)$ & $(2.4-30)$ & 43 & $2(4.7)$ & $(0.5-16)$ & 43 & $31(72.1)$ & $(56-84)$ & 43 & $7(16.3)$ & $(7-30)$ \\
\hline $2011 / 2012$ & 35 & $4(11.4)$ & $(3.2-26)$ & 46 & $0(0)$ & $(0-8)$ & 44 & $32(72.7)$ & $(57-85)$ & 44 & $10(22.7)$ & $(11-38)$ \\
\hline Total & 159 & & & 239 & & & 317 & & & 311 & & \\
\hline
\end{tabular}

Abbreviations: $\mathrm{Cl}$, confidence interval of the proportion; DS, decreased susceptibility; $\mathrm{R}$, resistant.

${ }^{*}$ Decreased susceptibility to cefixime and ceftriaxone defined by minimal inhibitory concentration (MIC) $>0.125 \mathrm{mg} / \mathrm{L}$. Ciprofloxacin resistance defined by MIC $>0.064 \mathrm{mg} / \mathrm{L}$, and penicillin resistance defined by MIC $>1.0 / \mathrm{L}$ or penicillinase-producing strains, according to the European Committee on Antimicrobial Susceptibility Testing (EUCAST). \#The 42 isolates from 1990 and the 66 isolates from 2000-2004 were stored at $-70^{\circ} \mathrm{C}$ and subcultured in 2005 for testing for penicillin and ciprofloxacin; and in 2011 for cefixime and ceftriaxone. The 36 strains from 2005/2006, the 85 strains from 2007/2008, the 43 strains from 2009/2010, and the 44 strains from 2011/2012 were tested under routine conditions for penicillin, ciprofloxacin and ceftriaxone. In 2011 the viable strains were tested for cefixime. By the end of 2011 , all strains were tested routinely for penicillin, ciprofloxacin, ceftriaxone and cefepime. This explains why the number of strains tested in each period differs for each antibiotic.

\section{Discussion}

In Switzerland, N. gonorrhoeae strains with decreased susceptibility to third-generation cephalosporins are emerging. We observed increasing rates of cefixime resistant strains, with a prevalence of 11\% between 2007 and 2012. Moreover, ceftriaxone MICs are rising. The proportion of isolates with elevated ceftriaxone MIC was $2.2 \%$ between 2007 and 2012 .

Our findings are in line with the worldwide documented decrease in cephalosporin susceptibility of $N$. gonorrhoeae. In the European Gonococcal antimicrobial susceptibility surveillance project (Euro-GASP), 9\% of isolates displayed decreased susceptibility to cefixime (MIC $\geq 0.25 \mathrm{mg} / \mathrm{L}$ ) in 2010. Cefixime resistance rates were $>5 \%$ in 11 of 21 European countries, and $>15 \%$ in 5 countries, including Austria, Denmark, Slovenia, Spain and Cyprus [22]. In the United States the percentage of cefixime resistant isolates ( $\mathrm{MIC} \geq 0.25 \mathrm{mg} / \mathrm{L}$ ) increased from $0.1 \%$ in 2006 to $1.4 \%$ in 2011 [23]. The rise was most pronounced in the West with resistance rates of $17 \%$ in Hawaii and $6.4 \%$ in San Diego, California, in 2011 [17].

These are in vitro data and clinical cephalosporin resistance has not yet been defined due to the fact that so far there have been too few documented treatment failures in order to establish a clear relationship between MIC and clinical treatment failure. In a recently published Canadian cohort study the rate of clinical treatment failure associated with isolates of $N$. gonorrhoeae with a cefixime MIC of $\geq 0.12 \mathrm{mg} / \mathrm{L}$ was $25.0 \%$ compared to $1.9 \%$ of isolates with a cefixime MIC of $<0.12 \mathrm{mg} / \mathrm{L}$ [9].

In 2008, we isolated one strain with a MIC for ceftriaxone of $0.25 \mathrm{mg} / \mathrm{L}$. No decreased susceptibility to ceftriaxone (MIC $\geq 0.25 \mathrm{mg} / \mathrm{L}$ ) was detected from 2004 to 2010 among Euro-GASP isolates. However, in correspondence to our data, ceftriaxone MICs increased from 2009 onwards [22]. In the United States the percentage of isolates with elevated ceftriaxone MICs $(\geq 0.125 \mathrm{mg} / \mathrm{L})$ rose slightly from $0 \%$ in 2006 to $0.4 \%$ in 2011 [17]. As displayed for cefixime, no

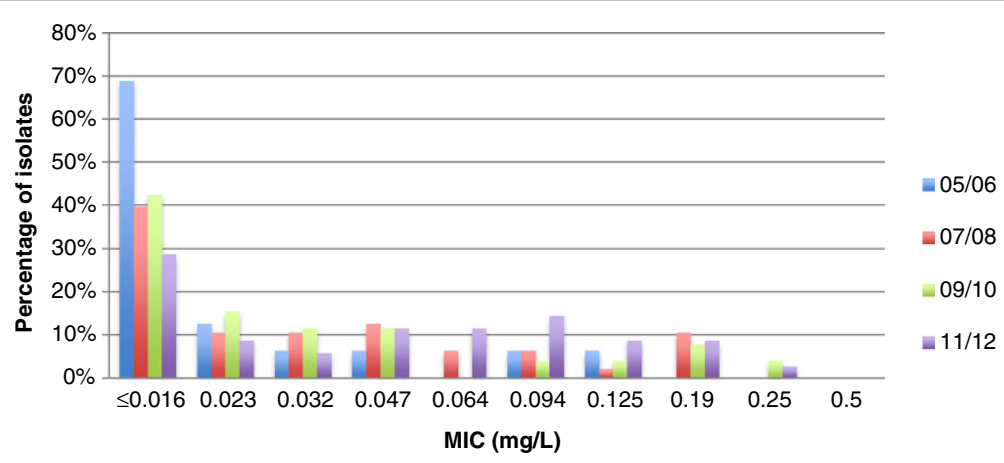

Figure 1 Distribution of minimum inhibitory concentrations of Neisseria gonorrhoea isolates for cefixime, 2005-2012. 2005/2006: 17 isolates. 2007/2008: 48 isolates. 2009/2010: 26 isolates. 2011/2012: 35 isolates. Abbreviation: MIC, minimum inhibitory concentration. 


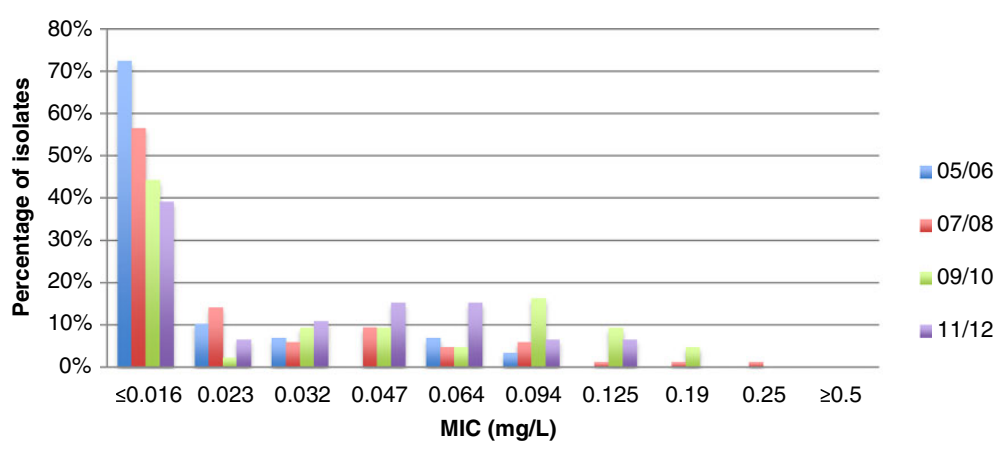

Figure 2 Distribution of minimum inhibitory concentrations of Neisseria gonorrhoea isolates for ceftriaxone, 2005-2012. 2005/2006: 29 isolates. 2007/2008: 85 isolates. 2009/2010: 43 isolates. 2011/2012: 46 isolates. Abbreviation: MIC, minimum inhibitory concentration.

threshold for resistance to ceftriaxone in $N$. gonorrhoeae has been defined yet. Increasing MICs for cephalosporins, however, may precede the emergence of resistance.

We found high ciprofloxacin resistance rates of $N$. gonorrhoeae, with $72.7 \%$ in 2012 . Our data demonstrates that during the 1990s and early 2000s, when ciprofloxacin was first choice for treatment, fluoroquinolone-resistant isolates emerged, and rapidly increased after 2000, correlating with the observation in other countries around the world [24-26]. By 2007, in the United States, and even earlier in other regions, including Europe and Australia, fluoroquinolones were no longer recommended for the treatment of gonococcal infections [27,28]. In Europe, ciprofloxacin resistance was 53\% (MIC $\geq 1 \mathrm{mg} / \mathrm{L})$ in 2010 [22], and 13.3\% in the United States in $2011(\geq 1 \mathrm{mg} / \mathrm{L})$ [23].

The World Health Organization (WHO) recommends discontinuation of empirical use of an antibiotic once 5\% of locally acquired gonococcal isolates are resistant [20]. The high rates of strains with decreased cefixime susceptibility and first isolates with elevated MICs for ceftriaxone shown by our data, support the recommendation of dual therapy for gonococcal infections with ceftriaxone plus either azithromycin or doxycycline. Combination regimens have been adopted as strategies for the treatment of other bacterial infections in the context of multidrug resistance development. The oral cephalosporin, cefixime, should no longer be used as first-line treatment.

In light of identified strains with decreased ceftriaxone susceptibility, health-care providers need to be vigilant for treatment failures, even in patients treated with recommended antibiotic regimens. In persons treated with alternative regimens, such as cefixime, test of cure is recommended. Continued local surveillance of gonococcal antimicrobial susceptibility is important. Due to the widespread use of nucleic acid amplification testing, culture for $N$. gonorrhoeae with antimicrobial susceptibility testing is not routinely done in clinical practice.

To the best of our knowledge, this is the first report on cephalosporin susceptibility testing of $N$. gonorrhoeae in
Switzerland. Longitudinal data on antimicrobial resistance in gonococci over a period of more than two decades was assessed. Nevertheless, the study has several limitations. The level of coverage was relatively low, attributed in a large part to the low number of gonococcal cultures taken routinely in clinical practice. The samples were not systematically collected within a sentinella system, which may result in selection of specimens with a higher resistance rate. Not all strains were tested for cephalosporins, which particularly concerns isolates obtained before 2006. However, the subset of $20-30 \%$ of all isolates stored, none showed decreased susceptibility for cefixime and ceftriaxone. It is therefore unlikely that we missed a relevant resistance signal. Finally, the isolates were not tested for azithromycin.

\section{Conclusions}

We found a high prevalence of $N$. gonorrhoeae with decreased susceptibility to cefixime, first strains with elevated MICs for ceftriaxone, and high resistance rates to ciprofloxacin. Treatment of gonorrhea with a combination regimen with ceftriaxone plus azithromycin or doxycycline is supported. Our data contributes to the global antimicrobial resistance surveillance of $N$. gonorrhoeae, and emphasizes the importance of further gonococcal antimicrobial resistance testing.

\section{Additional file}

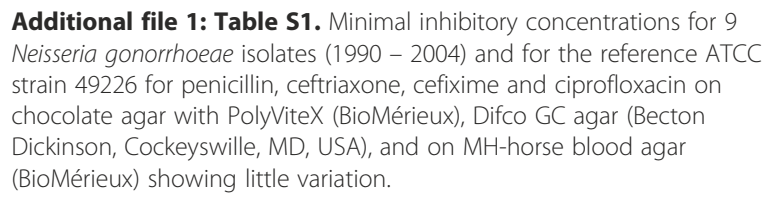

Competing interests

The authors declare that they have no competing interests.

\section{Authors' contributions}

HK, RZ and RW designed the study. SL and JM provided gonococcal isolates. MDM and RZ performed the laboratory analyses. All authors analysed and 
interpreted the data. HK and PH prepared the paper. All authors read and approved the final manuscript.

\section{Acknowledgements}

This study was supported in part by the University of Zurich. The authors thank J. Giger, R. Halter, G.Jost and the laboratory technicians for their dedicated help.

\section{Author details}

${ }^{1}$ Division of Infectious Diseases and Hospital Epidemiology, University Hospital Zurich, University of Zurich, Zurich, Switzerland. ${ }^{2}$ Institute of Medical Microbiology, University of Zurich, Zurich, Switzerland. ${ }^{3}$ Division of Dermatology, University Hospital Zurich, University of Zurich, Zurich, Switzerland.

Received: 23 September 2013 Accepted: 18 December 2013 Published: 26 December 2013

\section{References}

1. Marrazzo JM, Handsfield HH, Sparling PF: Neisseria gonorrhoeae. In Mandell, Douglas, and Bennett's Principles and Practice of Infectious Diseases. 7th edition. Edited by Mandell GL, Bennett JE, Dolin R. Philadelphia: Churchill Livingstone Elsevier; 2010.

2. Fleming DT, Wasserheit JN: From epidemiological synergy to public health policy and practice: the contribution of other sexually transmitted diseases to sexual transmission of HIV infection. Sex Transm Infect 1999, 75(1):3-17.

3. Stoltey JE, Barry PM: The use of cephalosporins for gonorrhea: an update on the rising problem of resistance. Expert Opin Pharmacother 2012, 13(10):1411-1420.

4. Yokoi S, Deguchi T, Ozawa T, Yasuda M, Ito S, Kubota Y, Tamaki M, Maeda S: Threat to cefixime treatment for gonorrhea. Emerg Infect Dis 2007, 13(8):1275-1277.

5. Lo JY, Ho KM, Leung AO, Tiu FS, Tsang GK, Lo AC, Tapsall JW: Ceftibuten resistance and treatment failure of Neisseria gonorrhoeae infection. Antimicrob Agents Chemother 2008, 52(10):3564-3567.

6. Unemo M, Golparian D, Syversen G, Vestrheim DF, Moi H: Two cases of verified clinical failures using internationally recommended first-line cefixime for gonorrhoea treatment, Norway, 2010. Euro Surveill 2010, 15(47).

7. Ison CA, Hussey J, Sankar KN, Evans J, Alexander S: Gonorrhoea treatment failures to cefixime and azithromycin in England, 2010. Euro Surveill 2011, 16(14).

8. Unemo M, Golparian D, Stary A, Eigentler A: First Neisseria gonorrhoeae strain with resistance to cefixime causing gonorrhoea treatment failure in Austria, 2011. Euro Surveill 2011, 16(43).

9. Allen VG, Mitterni L, Seah C, Rebbapragada A, Martin IE, Lee C, Siebert H, Towns L, Melano RG, Low DE: Neisseria gonorrhoeae treatment failure and susceptibility to cefixime in Toronto, Canada. JAMA 2013, 309(2):163-170.

10. Lewis DA, Sriruttan C, Muller EE, Golparian D, Gumede L, Fick D, de Wet J, Maseko V, Coetzee J, Unemo M: Phenotypic and genetic characterization of the first two cases of extended-spectrum-cephalosporin-resistant Neisseria gonorrhoeae infection in South Africa and association with cefixime treatment failure. J Antimicrob Chemother 2013, 68(6):1267-1270.

11. Tapsall J, Read P, Carmody C, Bourne C, Ray S, Limnios A, Sloots T, Whiley D: Two cases of failed ceftriaxone treatment in pharyngeal gonorrhoea verified by molecular microbiological methods. J Med Microbiol 2009, 58(Pt 5):683-687.

12. Unemo M, Golparian D, Hestner A: Ceftriaxone treatment failure of pharyngeal gonorrhoea verified by international recommendations, Sweden, 2010. Euro Surveill 2011, 16(6).

13. Ohnishi M, Golparian D, Shimuta K, Saika T, Hoshina S, Iwasaku K, Nakayama S, Kitawaki J, Unemo M: Is Neisseria gonorrhoeae initiating a future era of untreatable gonorrhea?: detailed characterization of the first strain with high-level resistance to ceftriaxone. Antimicrob Agents Chemother 2011, 55(7):3538-3545.

14. Unemo M, Golparian D, Nicholas R, Ohnishi M, Gallay A, Sednaoui P: Highlevel cefixime- and ceftriaxone-resistant Neisseria gonorrhoeae in France: novel penA mosaic allele in a successful international clone causes treatment failure. Antimicrob Agents Chemother 2012, 56(3):1273-1280.
15. Camara J, Serra J, Ayats J, Bastida T, Carnicer-Pont D, Andreu A, Ardanuy C: Molecular characterization of two high-level ceftriaxone-resistant Neisseria gonorrhoeae isolates detected in Catalonia, Spain. J Antimicrob Chemother 2012, 67(8):1858-1860.

16. Unemo M: The 2012 European guideline on the diagnosis and treatment of gonorrhoea in adults recommends dual antimicrobial therapy. Euro Surveill 2012, 17(47).

17. Update to CDC's Sexually transmitted diseases treatment guidelines, 2010: oral cephalosporins no longer a recommended treatment for gonococcal infections. MMWR Morb Mortal Wkly Rep 2012, 61(31):590-594

18. Bundesamt für Gesundheit. 2013. http://www.bag.admin.ch/ k_m_meldesystem/00733/00813/index.html?lang=de. Accessed May 4, 2013.

19. Le Lin B, Pastore R, Liassine N, Aramburu C, Sudre P: A new sexually transmitted infection (STI) in Geneva? Ciprofloxacin-resistant Neisseria gonorrhoeae, 2002-2005. Swiss Med Wkly 2008, 138(15-16):243-246.

20. World Health Organization (WHO): Global action plan to control the spread and impact of antimicrobial resistance in Neisseria gonorrhoeae. http://whqlibdoc.who.int/publications/2012/9789241503501_eng.pdf.

21. Breakpoint tablets for interpretation of MICs and zone diameters: version 3.1. European Committee on Antimicrobial Susceptibility Testing. http://www.eucast.org/clinical_breakpoints/. Accessed April 30.

22. Gonococcal antimicrobial susceptibility surveillance in Europe 2010 Stockholm: ECDC 2012:European Centre for Disease Prevention and Control (ECDC). Available from: http://ecdc.europa.eu/en/publications/Publications/1206Gonococcal-AMR.pdf.

23. CDC Grand rounds: the growing threat of multidrug-resistant gonorrhea. MMWR Morb Mortal Wkly Rep 2013, 62(6):103-106.

24. Fenton KA, Ison C, Johnson AP, Rudd E, Soltani M, Martin I, Nichols T, Livermore DM: Ciprofloxacin resistance in Neisseria gonorrhoeae in England and Wales in 2002. Lancet 2003, 361(9372):1867-1869.

25. Tanaka M, Nakayama H, Notomi T, Irie S, Tsunoda Y, Okadome A, Saika T, Kobayashi I: Antimicrobial resistance of Neisseria gonorrhoeae in Japan, 1993-2002: continuous increasing of ciprofloxacin-resistant isolates. Int J Antimicrob Agents 2004, 24(Suppl 1):S15-22.

26. Hsueh PR, Tseng SP, Teng LJ, Ho SW: High prevalence of ciprofloxacinresistant Neisseria gonorrhoeae in Northern Taiwan. Clin Infect Dis 2005, 40(1):188-192.

27. Update to CDC's sexually transmitted diseases treatment guidelines, 2006: fluoroquinolones no longer recommended for treatment of gonococcal infections. MMWR Morb Mortal Wkly Rep 2007, 56(14):332-336.

28. Tapsall JW, Limnios EA, Murphy D: Analysis of trends in antimicrobial resistance in Neisseria gonorrhoeae isolated in Australia, 19972006. J Antimicrob Chemother 2008, 61(1):150-155.

\section{doi:10.1186/1471-2334-13-603}

Cite this article as: Kovari et al:: Decreased susceptibility of Neisseria gonorrhoeae isolates from Switzerland to Cefixime and Ceftriaxone: antimicrobial susceptibility data from 1990 and 2000 to 2012. BMC Infectious Diseases 2013 13:603.

\section{Submit your next manuscript to BioMed Central and take full advantage of:}

- Convenient online submission

- Thorough peer review

- No space constraints or color figure charges

- Immediate publication on acceptance

- Inclusion in PubMed, CAS, Scopus and Google Scholar

- Research which is freely available for redistribution 